\title{
RUPTURA ESPONTÁNEA DEL TENDÓN DE AQUILES EN PACIENTE J OVEN SIN ANTECEDENTES PATOLÓGICOS
}

Francisco Javier García M D*, Rafael A. B runicardi M D**, Francis K. Graziano M D*** , David M arulanda M D**, José Fernando Polo M D****

\section{Resumen}

La ruptura del tendón de Aquiles representa el $20 \%$ de todas las lesiones de tendones largos del cuerpo humano. La lesión espontánea es rara y se relaciona con el uso crónico de corticoides y fluorquinolonas, insuficiencia renal crónica y enfermedades del colágeno. La dorsiflexión forzada con el pie en plantiflexión es el mecanismo más frecuente, siendo el resultado de una fuerza de contracción excéntrica de los gastrosóleos combinada con las fuerzas de reacción al piso. Consideramos de importancia el reporte de este caso por ser un paciente de 22 años de edad con ruptura espontánea y estudios histopatológicos, que contrasta con lo reportado en la literatura.

Palabras clave: tendón de Aquiles, ruptura espontánea, lesión tendinosa.

Abreviaturas: TA, tendón de Aquiles.

\section{SPONTANEOUS ACHILLES TENDON RUPTURE IN A YOUNG HEALTHY INDIVIDUAL}

\begin{abstract}
Achilles tendon ruptures account for $\mathbf{2 0 \%}$ of all large tendon ruptures. A spontaneous lesion is unusual and is secondary to long term corticosteroid therapy and usage of fluoroquinolones, chronic renal failure and collagen deficiencies. The most common mechanism of injury includes excessive dorsiflexion of a plantar-flexed foot resulting from an eccentric load of the gastrosoleus muscles combined with ground-reaction forces. Our case report is of interest because it is a 22-year-old male patient who presented a spontaneous rupture and histopathology studies which contrast with those described in the literature.
\end{abstract}

Key words: Achilles tendon; spontaneous rupture; tendon injuries.

Fecha recibido: junio 18 de 2014 - Fecha aceptado: octubre 17 de 2014

* Ortopedista y traumatólogo. Especialista en pie y tobillo, Hospital Infantil Universitario de San José. Bogotá DC, Colombia.

** Residente IV de Ortopedia y Traumatología. Fundación Universitaria de Ciencias de la Salud. Bogotá DC, Colombia.
*** Residente III de Patología. Fundación Universitaria de Ciencias de la Salud. Bogotá DC, Colombia.

***** Patólogo.Jefe del Servicio de Patología del Hospital Infantil Universitario de San José. Instructor Asociado, Fundación Universitaria de Ciencias de la Salud. Bogotá DC, Colombia. 


\section{Introduc ción}

A pesar de que el tendón de A quiles es el más fuerte del cuerpo humano ${ }^{1}$, es el que con más frecuencia se ve afectado en los miembros inferiores, representando el $20 \%$ de todas las lesiones de tendones largos. ${ }^{2}$ La lesión espontánea es rara y a menudo se relaciona con el uso crónico de corticoides y fluorquinolonas, aumentando dicho riesgo cuando se prescriben en forma simultánea. ${ }^{3} \mathrm{~A}$ demás, está relacionada con enfermedades del colágeno e insuficiencia renal crónica.

En pacientes sin antecedente de consumo de esteroides ni enfermedad sistémica, la etiología es incierta. Cetti y colaboradores ${ }^{4}$ evaluaron clínica e histopatológicamente sesenta casos consecutivos de rupturas espontáneas en pacientes con edades comprendidas entre 18 y 60 años, obteniendo como conclusiones que la degeneración y necrosis del TA roto no solo ocurre en el sitio de la ruptura, sino que lo afecta en su totalidad; el tendón contralateral asintomático, sufre en menor grado los mismos cambios degenerativos evidenciados en el roto, como es la inflamación aguda en el lado de la ruptura, a diferencia del contral ateral. Sugieren que el daño difuso del TA ocurre debido a microtraumas a repetición y sobreuso, favoreciendo la degeneración del colágeno y la necrosis del tenocito. Si éste fenómeno continúa en el tiempo, desencadena una respuesta inflamatoria aguda que puede debilitar al tendón hasta el punto en que no resiste fuerzas mecánicas durante el ejercicio y falla. ${ }^{4} \mathrm{~L}$ a ruptura espontánea del TA también se reporta en individuos sanos, activos, entre 30 y 50 años de edad, siendo más frecuente en hombres. ${ }^{5}$ Sin embargo, en la literatura, no existen reportes ni series de casos en pacientes menores de 30 años.

A Igunos autores recomiendan el tratamiento quirúrgico para la rehabilitación rápida y lograr restaurar la máxima función del tendón y de la extremidad. ${ }^{6}$ Nilsson-H elander, y col. ${ }^{6}$ no encontraron diferencia estadística significativa entre el tratamiento quirúrgico y el ortopédico en las lesiones agudas del TA, pero coinciden con $M$ yerson en que la movilización precoz es beneficiosa para la rehabilitación. ${ }^{7}$

\section{Reporte de caso}

Hombre de 22 años estudiante de medicina, de contextura y estatura promedio para la población colombiana, que durante actividad deportiva en la fase de despegue de la marcha, refirió dolor súbito sobre la parte posterior del tobillo izquierdo, teniendo sensación de haber sido "golpeado con una piedra", con persistencia del dolor e imposibilidad para la bipedestación. Consultó a urgencias por dolor, edema y limitación funcional del tobillo izquierdo. Presentaba signo de Thompson positivo para lesión y defecto pal pable en la sustancia del tendón (Figura 1) siendo diagnosticada la ruptura del TA. Se manejó con inmovilización en equino de 10 con férula de yeso, se formularon medidas antiedema y se programó para manejo quirúrgico. El paciente y su madre niegan antecedentes patológicos, consumo de corticoides ni de quinolonas, sólo refieren que el paciente fue manejado con trimetoprim/sulfametoxazol durante cerca de seis meses cuando tenía dos años de edad por infección respiratoria.

A los diez días se practicó cirugía reconstructiva mediante técnica percutánea (Figura 2) y sutura Ethibond ${ }^{\mathrm{TM}}$ 0 , con la que se reportan resultados similares a los logrados con cirugía abierta, obteniendo mejor apariencia cosmética, menor incidencia de complicaciones en la herida y sin aumentar el riesgo de rerruptura. ${ }^{8}$ Como hallazgos intraoperatorios se observó el TA desflecado y friable, lográndose afrontar en forma satisfactoria ambos extremos del tendón y consiguiendo una adecuada

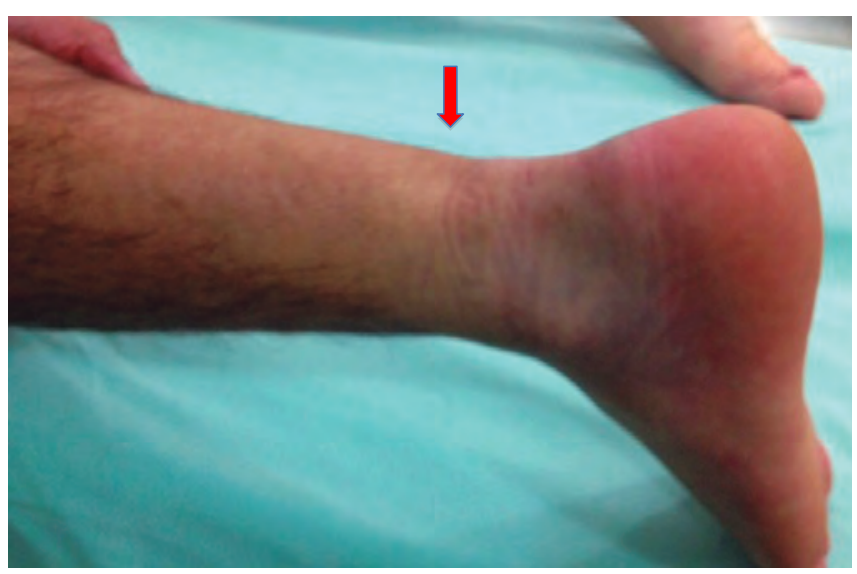

Figura I. Se evidencia defecto sobre la sustancia del tendón de Aquiles izquierdo. 
reconstrucción y tensión con respecto a la extremidad contralateral previamente preparada. Con autorización del paciente se toma biopsia del sitio de la lesión del TA así como del tendón contralateral, para ser evaluados en conjunto por el servicio de patología del Hospital Infantil Universitario de San J osé de Bogotá DC, Colombia. Se inmoviliza con férula en equino fisiológico del tobillo durante dos semanas, luego de las cuales se valoró, encontrando heridas cicatrizadas, se retiraron puntos y se cambió la inmovilización por ortesis articulada de tobillo tipo Walker bloqueado en $5^{\circ}$ de equino, con plantiflexión activa por dos semanas sin permitir apoyo. Dos semanas después se graduó el Walker para permitir dorsiflexión hasta $0^{\circ}$ y plantiflexión activa, se inició apoyo progresivo y se inició fisioterapia con restricciones. A la sexta semana de tratamiento se permitió rango fisiológico de la dorsiflexión protegiendo el apoyo con el Walker hasta completar doce semanas, luego de lo cual se suspendió la inmovilización continuando con la rehabilitación en fisioterapia. L uego de seis meses de posoperatorio el paciente inició actividades deportivas recreacionales en forma progresiva, logrando llegar al mismo nivel que tenía antes de la lesión. Después de catorce meses de haberse realizado el procedimiento, el paciente se encuentra realizando sus actividades cotidianas y deportivas recreativas sin restricciones, con adecuada potencia para la plantiflexión (Figura 3).

\section{Disc usión}

L a ruptura espontánea del TA ha sido reportada como complicación del tratamiento crónico con corti-

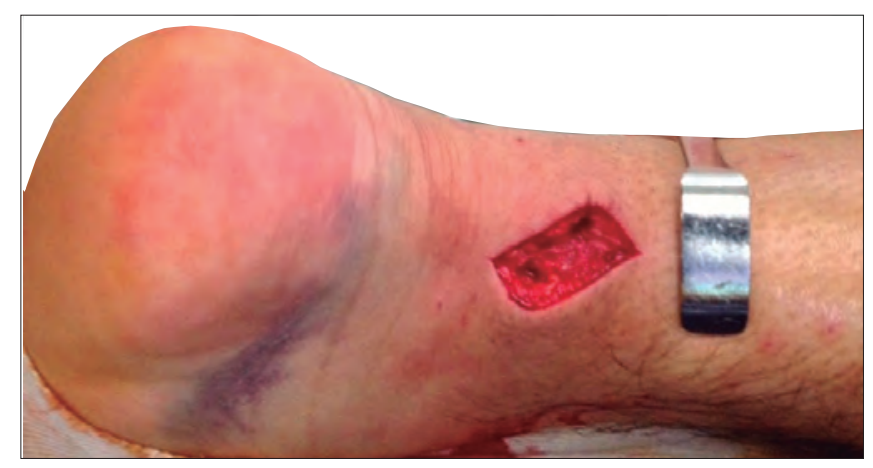

Figura 2. Reconstrucción del tendón de Aquiles izquierdo con técnica percutánea. Como referencia, se observa separador de Farabeuf.

coesteroides, también existen reportes que sugieren predisposición en pacientes con hipercolesterolemia. L a ruptura espontánea del TA en pacientes jóvenes suele ocurrir a una edad media de 38 años, pero no existen reportes en la literatura en menores de 30 años. Es probable que el mecanismo en este caso fue producto de una dorsiflexión forzada con un pie en plantiflexión, resultado de una fuerza de contracción excéntrica de los gastrosól eos combinada con las fuerzas de reacción al piso, lo que inicia el punto de falla para la ruptura del TA . Habusta9 reporta rupturas espontáneas en tendones degenerados y con debilidad de la estructura de colágeno, que puede ser ocasionado por tratamiento crónico con corticoesteroides, por hipercolesterolemia o hipotiroidismo, lo que disminuye la vascularidad del tendón y en forma secundaria reduce su fuerza. Los resultados de histopatología (Figura 4) de este caso evidencian un proceso inflamatorio sin cambios
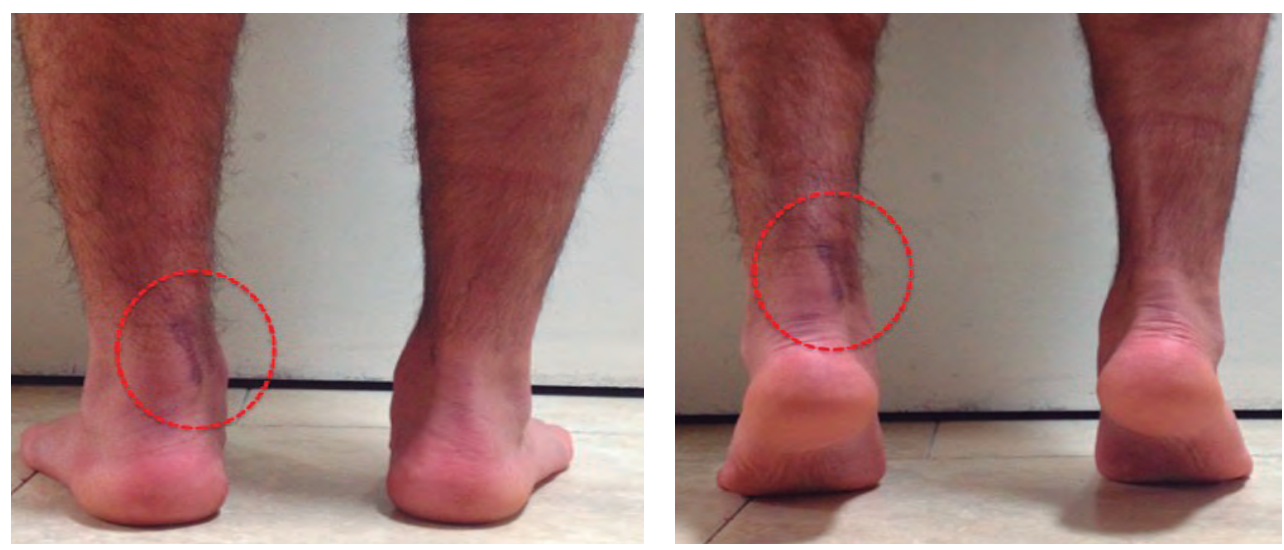

Figura 3. A los catorce meses de posoperatorio realiza sus actividades en forma cotidiana. En el círculo, se evidencia la cicatriz quirúrgica. 


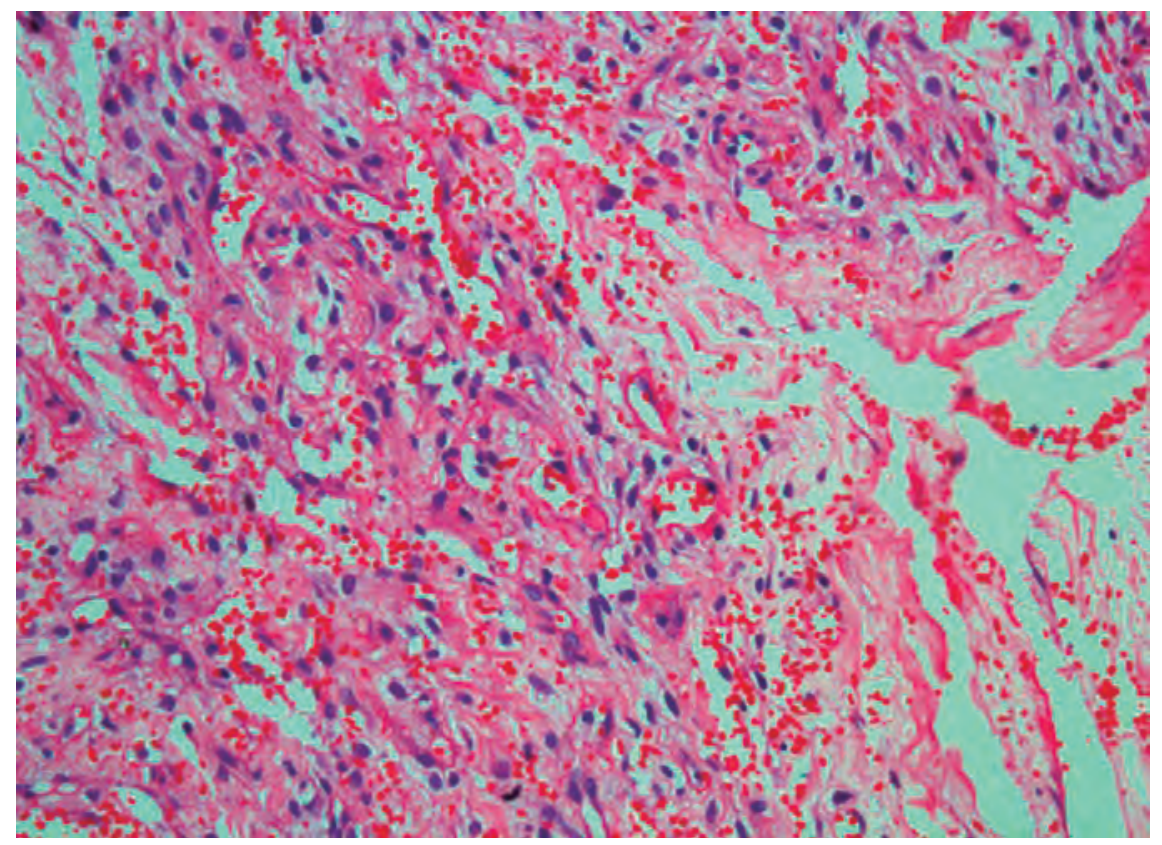

Figura 4. Proliferación de estructuras vasculares de aspecto reparativo con presencia de ocasionales neutrófilos. (HE 20x).

del tendón contralateral. No hay patología previa del tendón ni predisposición, en contraste con lo reportado en la literatura. ${ }^{4}$

\section{Referencias}

1. Young JS, Movin T, Maffulli N. Achilles Tendon Rupture Generalities. In: M affulli N, A Imekinders LC, editors. The A chilles Tendon. London: SpringerVerlag; 2007. p. 141-50.

2. SK Rao, BC Navadgi, A Vasdev: Bilateral spontaneous rupture of A chilles tendons: A case report. J Orthop Surg (Hong Kong). 2005; 13(2):178-80.

3. Khanzada Z, Rethnam U, Widdowson D, M irza A. Bilateral spontaneous nontraumatic rupture of the A chilles tendon: a case report. J Med Case Reports. $2011 ; 5: 263$

4. Cetti R, Junge J, Vyberg M. Spontaneous rupture of the A chilles tendon is preceded by widespread and bilateral tendon damage and ipsilateral inflammation: a clinical and histopathologic study of 60 patients. Acta Orthop Scand. 2003; 74(1):78-84.

5. Hashin Z, Dahabreh Z, Bin Jemain MT, Williams HR. Bilateral simultaneous achilles tendon rupture in the absence of risk factors. Foot Ankle Spec. 2011; 4(3):175-8.

6. Nilsson-Helander $K$, Silbernagel $K G$, Thomeé $R$, Faxén $E$, Olsson $N$, Eriksson $\mathrm{BI}$, et al. A cute achilles tendon rupture: a randomized, controlled study comparing surgical and nonsurgical treatments using validated outcome measures. A m J Sports M ed. 2010; 38(11):2186-93.

7. Myerson MS. Disorders of the A chilles Tendon. In: M yerson MS, editors. Reconstructive foot and ankle surgery : management of complications. 2nd ed. Philadelphia, PA : Elsevier/Saunders; 2010 .p. 331-48.

8. Henríquez $H, M$ uñoz $R, C$ arcuro $G, B$ astías $C$. Is percutaneous repair better than open repair in acute A chilles tendon rupture?. Clin Orthop Relat Res. 2012; 470(4):998-1003.

9. Habusta SF. Bilateral simultaneous rupture of the A chilles tendon. A rare traumatic injury. Clin Orthop Relat Res. 1995; (320):231-4. 